\title{
AI Power Hand Gesture Control Vehicle
}

\author{
Ms. Vidhi Kalpesh Sheth \\ Information Technology Department Shah and Anchor \\ Kutchhi Engineering College \\ Mumbai, India
}

Ms. Vrushali Kalpesh Sheth

Information Technology Department Shah and Anchor Kutchhi Engineering College

Mumbai, India

\begin{abstract}
There are lots of technologies or techniques emerging in the auto vehicles sector. So being in 21 st century the Artificial Intelligence and the Internet of Things has given a boost to all these sectors being related to the auto-vehicles. Earlier, the robotics vehicles was being controlled via wired cable or a bluetooth device which had a lot of drawback, due to which it ensures less compatibility and also the vehicles in real world application such as military was not having that impact of Artificial Intelligence or Internet of things due to which there were more a lot of efforts or human task had to be undertaken. In this work, detailed survey and comparative analysis of the robotic vehicle is being presented[8]. Most of the systems, use IOT and AI which reduces human efforts, increased compatibility, ensure security and less human interaction required. Some of the robotic vehicles were controlled wirelessly via hand gesture even at very remote areas. Such type of vehicles can also be considered in real world application like military/defense, surveillance at the border and similarly during the war. If such type of systems is implemented with the power of AI, it would help to detect the enemy and notify the user.
\end{abstract}

Keywords-Artificial intelligence; Gesture control vehicle; Hand gesture recognition; Robotic car

\section{INTRODUCTION}

A Gesture Controlled Vehicle is a kind of vehicle which can be controlled by the user's hand gesture not by old buttons. Human hand gestures are natural and with help of wireless communication[1]. It is easier to interact with vehicle in a friendly way than usual remote-controlled vehicle. In fig 1 it shows that the vehicle moves according to your hand movement from a distance. The vehicle moves forward, backward, right and left respectively. The vehicle stops when the hand is kept parallel to ground. There is a camera mounted on vehicle which is capable of zooming in and out as well as capable of rotating 360 degrees. The output video will stream constantly on the android based smart phone or laptop[5]. The structure of Vehicle uses Arduino Uno, RF transmitter, receiver module, accelerometer, GPS, webcam. The Arduino Uno reads analog output values i.e. $\mathrm{X}$ - axis and $y$-axis values of accelerometer which changes depending on hand motion and converts that analog value to respective digital values[3]. The digital values are processed by microcontroller and send the command to vehicle via RF transmitter. This command are received by $\mathrm{RF}$ receiver and processed by microcontroller to drive the motor to a particular direction[6].

\author{
Ms. Bhakti Kiran Rathod \\ Information Technology Department Shah and Anchor \\ Kutchhi Engineering College \\ Mumbai, India \\ Dr. Kranti Vithal Ghag \\ Information Technology Department Shah and Anchor \\ Kutchhi Engineering College \\ Mumbai, India
}

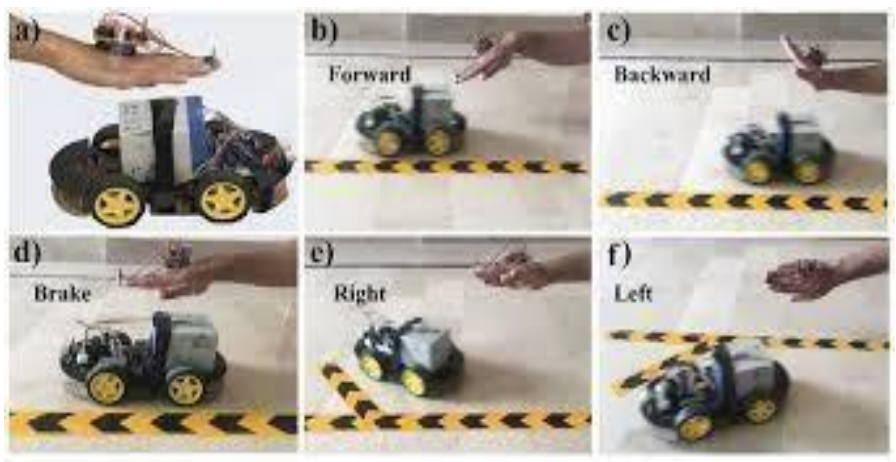

Fig 1: Hand Movements to control Car

\section{LITERATURE SUREVEY}

A. The first paper surveyed is "(2019) Deep Learning Algorithm Using Virtual Environment Data for Self Driving Car'"[1]. The approaches of this paper were an autonomous driving technique announced by NVidia using car games. In this paper they had used different datasets such as "Learning data with an end to end method". The performance of this paper is about $80 \%$. The Precision and Recall of this paper is about $81 \%$ and $85 \%$.

B. The second paper surveyed is "(2006) A Cognitive agent based approach to varying behaviors in computer generated forces system to model Scenarios

like coalitions"[2]. The approach of this paper is Dynamic Variation in behavior of entities in military based computer generated forces system scenarios. The datasets which they were used is Methodology based on the application of Autonomous cooperative building blocks in order to handle the CGF. The performance, precisions and recall of this paper is $77 \%, 74 \%$ and $73 \%$.

C. The third paper surveyed is "(2017) Military based vehicle to greed and vehicle to vehicle micro greed system architecture and implementation"[3] This paper describes a real-life military use of the Vehicle-to- Grid / Vehicle-to-Vehicle (V2G-V2V) based micro grid network. The device offers a plug-and-play, very quick forming, and smart, aggregated, and effective power solution for a military base contingency that can be set up in less than 20 minutes and is ready to produce up to $240 \mathrm{~kW}$ of 3 -phase (3Ø) 208Y/120VAC. The system uses TransmissionIntegrated Generators (TIGs) vehicles to produce 600VDC power for vehicle hotel loads (i.e. electrification of nonpropulsion and auxiliary loads) and off-board loads (tents / shelters, contact centers or other electrical loads). This 
initiative includes four military combat vehicles-two M1152 HMMWV vehicles fitted with $30 \mathrm{~kW}$ of on-board vehicle power (OBVP) And two MaxPro Dash MRAP vehicles fitted with $120 \mathrm{~kW} 3000$ Transmission- Integrated Generators (3TIGs) with V2 G and V2V capacity, four $60 \mathrm{~kW}$ DC-to-3 $\varnothing$ AC power converters with 600 VDC bus distribution systems and four 22,8 kWh of Energy Storage Units (ESU). The performance, precision and recall of this paper are $70 \%, 75 \%$ and $65 \%$.

D. The fourth paper surveyed is "(2016) Probabilistic risk based security assessment of power system

considering incumbent threat and uncertainties"[4]. Indepth Power Systems (PS) security analyzes includes consideration of vulnerabilities to natural and humanrelated threats that can trigger multiple dependent continent genes. At the other hand, these events also lead to high impact on the system, so it can become difficult to make decisions aimed at improving protection. The risk associated with each contingency may be the introduction of uncertainty. The performance, precision and recall of this paper are $69.50 \%, 80 \%$ and $75 \%$.

E. The fifth paper surveyed is “(2019) Design and implementation of hand movement's controlled robotic vehicle with wireless live streaming feature"'[5]. This paper describes the design and implementation of a robotic vehicle that can be operated from all directions using a wireless camera mounted at the top of the vehicle to relay live video streaming to the end of the user. This thus avoids the hassle of gesture recognition and image processing software, or even the use of switches or joysticks to guide a robot's movement in various directions, and provides the user with a wireless monitoring facility. They have design a robotic controlled vehicle wirelessly. Person can control the movement in forward direction, backward direction, left and right direction by only using the hand movement gesture. Also this system has use on board camera to provide facility from remote places. The performance, precision and recall are $84 \%, 86 \%$ and $91 \%$.

F. The sixth paper surveyed is "(2019) Smart glove and hand gesture based control interface for multipolar

aerial vehicles"[6]. This project's research objective performs a comparative performance analysis between realtime image processing and object recognition of Artificial Intelligence while implementing an autonomous wheelchair obstacle avoidance device. The proposed framework for the identification of obstacles is accomplished by applying camera sensor with the application of Artificial Intelligence techniques in image processing. In designing the object recognition algorithm, a pre-trained Con- volitional Neural Network model known as Mobile Net SSD and Deep Neural Network (DNN) module in the Open CV library (for live video streams) are used. The performance, precision and recall of this project are $89.79 \%, 60 \%$ and $85 \%$.

G. The seventh paper surveyed is "(2017) Study of Evaluation method of in vehicle gesture control" [7]. In a simulated driving cockpit with an Eye-Tracker involved, a usability test for in-vehicle gesture control was performed.
In the study, 14 usability- related data types were collected from which 11 usability-related indexes were chosen to construct a Fuzzy

Comprehensive Evaluation System. Through this method, the most appropriate gesture solution was identified for each task, which can provide reference for the future application of gesture control inside the vehicle. The performance, precisions and recall of this paper are $75 \%$, $34 \%$ and $70 \%$.

H. The eight paper surveyed is "(2018)

Development of intelligent riding comfort monitoring system for

automated vehicle"[8]. This paper demonstrates the conceptual framework for reflection to the real driving power of the intelligent riding comfort monitoring system. The experiment is structured for the precise analysis of human behavior. In certain types of automated vehicles, the proposed scheme will theoretically be extended to enhance the perceived efficiency of automated driving. The performance, precisions and recall of this paper are $68 \%$, $80 \%$ and $78 \%$.

I. The ninth paper surveyed is "(2018) Wrist Cam: A wearable sensor for hand trajectory gesture recognition and intelligent human - robot

interaction". A wearable wrist-worn camera sensor (Wrist Cam) was shown here to identify movements of the hand trajectories. The user's hand's moving velocity was deduced from the corresponding Speeded up Robust Features (SURF) key points of the video sequence's moving history. In addition, continuous gesture segmentation was accomplished by detecting the predefined gesture starting signal from the image's hand field, which was segmented by the Lazy Snapping algorithm. In their research, the Dynamic Time Warping (DTW) algorithm identified 10 types of gestures and 1350 gesture samples collected from 15 subjects at 3 separate scenes, and the results achieved an overall accuracy of identification up to 97.6 percent. However, by guiding a cooperative robot to draw letters on paper, the practicability of the proposed device was further demonstrated. The performance, precision and recall of this paper are $79 \%, 68 \%$ and $65 \%$. 
III.

COMPARATIVE ANALYSIS

Table 1: Comparative Analysis of technique for Hand Gesture Controlled Vehicl[1] [2] [3] [4] [5] [6] [7] [8]

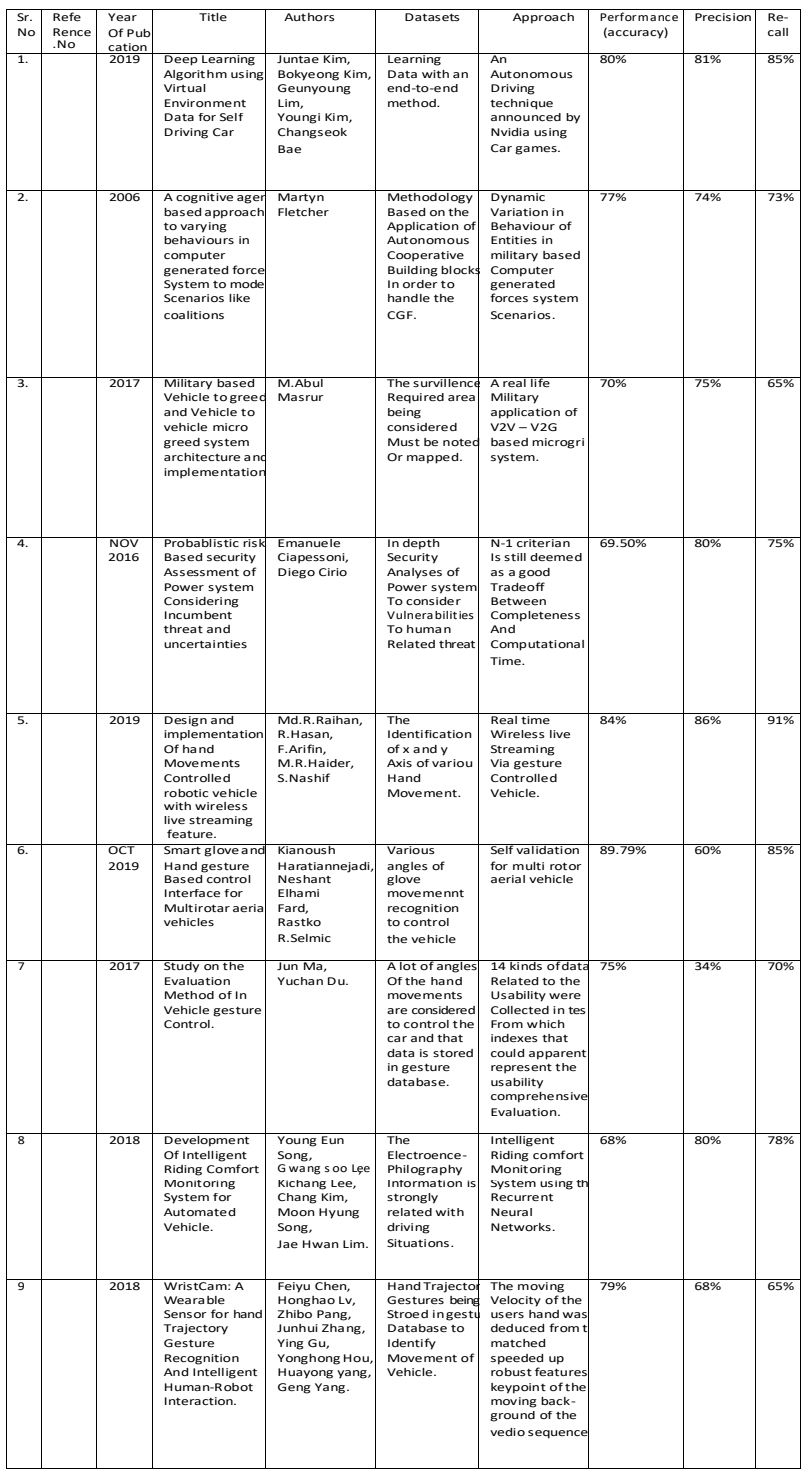

\section{DISCUSSION}

Surveyed gesture-controlled vehicles were compared on the basis of accuracy, precision and recall in Table 1 . Smart glove and hand gesture-based control interface for multirotor aerial vehicles with the dataset of studying the various angles of glove movement recognition to control the vehicle achieved a remarkable accuracy of $89.79 \%$ is. The approach is used to satisfy the speed requirements. The hand gesture recognition with aerial vehicles is robust to scale changes as compared to other literature reviewed[6].

\section{CONCLUSION}

Now-a-days the demand for Artificial Intelligence based military vehicles has increased tremendously. In future we can extend the range of the vehicle by using the satellite. In fig 2 the gesture controlled systems gives an alternative way of controlling robots. With an advance in the AI a variety of features can be added to the Gesture Controlled Vehicle that can be used in various sector like defense, surveillance, industries, hospitals, etc. This technology or technique can be used to give a boost to the manufacturing of the vehicles and giving its contribution to the automobile sectors. It is very efficient, compatible and easy to use. By converting this robotic car to an amphibious vehicle which can work on land as well as water and increases the range of its application. In future the similar concept can be used in robotic ARM making it controlled via wirelessly using Gesture.

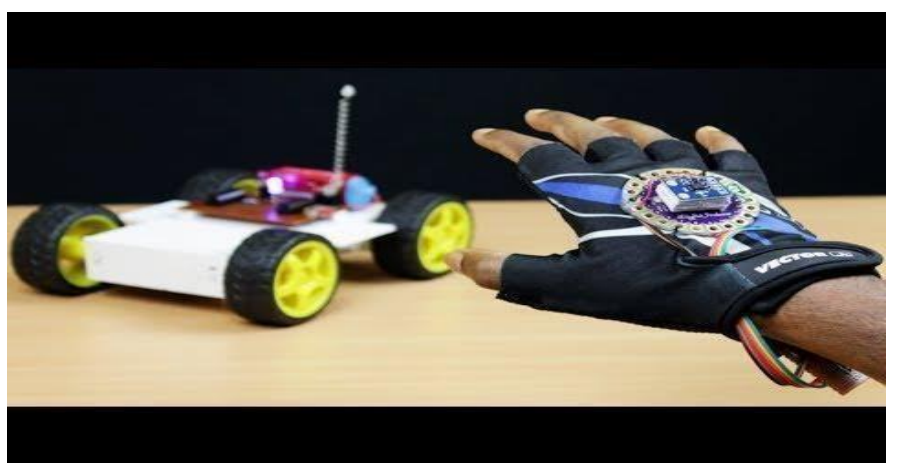

Fig 2: Gesture controlled vehicle

\section{REFERENCES}

[1] M. R. Raihan, R. Hasan, F. Arifin, S. Nashif, and M. R. Haider "Design and Implementation of a Hand Movement Controlled Robotic Vehicle with Wireless Live Streaming Feature," 2019 IEEE International Conference on System, Computation, Automation and Networking (ICSCAN), 2019.

[2] Jun Ma and Yuchun Du, "Study on the Evaluation Method of InVehicle Gesture Control," 2017 IEEE 3rd International Conference on Control Science and Systems Engineering.

[3] M. A. Masrur, A. G. Skowronska, J. Hancock, S. W. Kolhoff, D. Z. Mcgrew, J. C. Vandiver, and J. Gatherer, "Military-Based Vehicle-to- Grid and Vehicle-to-Vehicle Micro grid-System Architecture and Implementation," IEEE Transactions on Transportation Electrification, vol. 4, no. 1, pp. 157-171,2018.

[4] E. Ciapessoni, D. Cirio, G. Kjolle, S. Massucco, A. Pitto, and M. Sforna, "Probabilistic risk-based security assessment of power systems considering incumbent threats and uncertainties," 2017 IEEE Power \&amp; Energy Society General Meeting, 2017

[5] Yoon Lee, Shang Tan, Yeh Goh, Chern Lim. 2019 IEEE 3rd Advanced Information Management, Communicates, Electronic and Automation Control Conference (IMCEC) - 2019.

[6] Young Eun Song, Chang Il Kim, Moon Hyung Song, Gwang Soo Lee, Jae Hwan Lim, Kichang Leeand Moon Sik Kim, "Development of Intelligent Riding Comfort Monitoring System for Automated Vehicle,’2018.

[7] L. Jiang, M. Xia, X. Liu, and F. Bai, "Givs: Fine-Grained Gesture Control for Mobile Devices in Driving Environments," IEEE Access, vol. 8, pp. 49229-49243, 2020.

[8] Feiyu Chen, Honghao Lv, Zhibo Pang, Member, IEEE, Junhui Zhang, Yonghong Hou, Ying Gu, Huayong Yang, Member, IEEE, and Geng Yang, WristCam: A Wearable Sensor for Hand Trajectory Gesture Recognition and Intelligent Human-Robot Interaction. , 2018 IEEE. 